\title{
Fractal time random walk and subrecoil laser cooling considered as renewal processes with infinite mean waiting times
}

\author{
F. Bardou \\ IPCMS, CNRS and Université Louis Pasteur 23 rue du Loess, BP 43, F-67034 Strasbourg Cedex 2, Franc®
}

\begin{abstract}
There exist important stochastic physical processes involving infinite mean waiting times. The mean divergence has dramatic consequences on the process dynamics. Fractal time random walks, a diffusion process, and subrecoil laser cooling, a concentration process, are two such processes that look qualitatively dissimilar. Yet, a unifying treatment of these two processes, which is the topic of this pedagogic paper, can be developed by combining renewal theory with the generalized central limit theorem. This approach enables to derive without technical difficulties the key physical properties and it emphasizes the role of the behaviour of sums with infinite means.
\end{abstract}

To appear in: Proceedings of Cargese Summer School on "Chaotic Dynamics and Transport in Classical and Quantum Systems", August 18-30 (2003).

\section{Introduction}

The fractal time random walk [1, 2] has been developed in the 1970's to explain anomalous transport of charge carriers in disordered solids. It describes a process in which particles jump from trap to trap as a result of thermal activation with a very broad (infinite mean) distribution of trapping times. It results in an unusual timedependence of the position distribution which broadens while the peak remains at the origin. The method of choice to study the fractal time random walk is the continuous time random walk technique.

Subrecoil laser cooling 3, 4] has been developed in the 1990 's as a way to reduce the thermal momentum spread of atomic gases thanks to momentum exchanges between atoms and laser photons. It is a process in which, as a result of photon scattering, atoms jump from a momentum to another one with a very broad distribution of waiting times between two scattering events. It results in an unusual time dependence of the momentum distribution which narrows without fundamental limits hence giving access to temperatures in the nanokelvin range. The method of choice to study subrecoil laser cooling is renewal theory [5].

Fractal time random walks and subrecoil cooling seem at first sight very dissimilar. The first mechanism generates a broader and broader distribution, while the second generates a narrower and narrower distribution. Nevertheless, inspection of the theories of both phenomena reveals strong similarities: the continuous time random walk and the renewal theory are two closely related ways to tackle related stochastic processes. Physically, the two mechanisms share a common core, a jump process with a broad distribution of waiting times.

The aim of this pedagogic paper is to bridge the gap

*Electronic address: bardou@ipcms.u-strasbg.fr between fractal time random walk and subrecoil laser cooling. We show that the essential results of the two theories can be obtained nearly without calculation by combining the simple probabilistic reasoning underlying renewal theory and the generalized central limit theorem applying to broad distributions. This provides more direct derivations than in earlier approaches, at least for the basic cases considered here.

In the first part, we describe the microscopic stochastic mechanisms at work in the fractal time random walk and in subrecoil cooling and relate them to renewal processes. In the second part, we explain elementary properties of renewal theory and derive asymptotic results using the generalized central limit theorem and Lévy stable distributions. In the third part, we draw the consequences for the fractal time random walk and subrecoil cooling. The fourth part contains bibliographical notes.

\section{FRACTAL TIME RANDOM WALK AND SUBRECOIL COOLING: MICROSCOPIC MECHANISMS}

\section{A. Fractal time random walk}

The notion of fractal time random walk emerged from the observation of unusual time dependences in photoconductivity transient currents flowing through amorphous samples. It can be schematized in the following way.

Consider first a one dimensional situation called the Arrhenius cascade [6] in which the charge carriers are placed in a random potential with many local wells and barriers and can jump from one well to another one thanks to thermal activation (Fig. 1 $\mathrm{k}$ ). The Arrhenius cascade potential presents two features: a global tilt representing the effect of the electric field on the carriers and local random oscillations creating metastable traps separated by barriers representing the disorder created by the amorphous material. Thus the potential seen by the carriers is a kind of random washboard with a discrete number of metastables states.

The mean lifetime of state $i$, i.e., the mean waiting time before the occurrence of a thermal jump, is given 


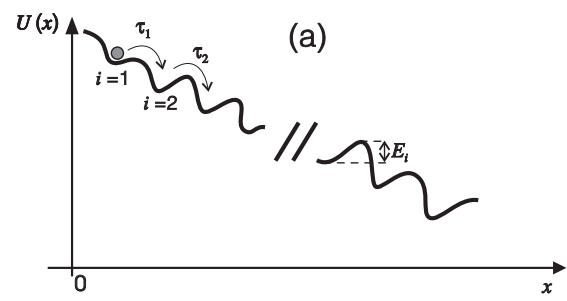

(b)

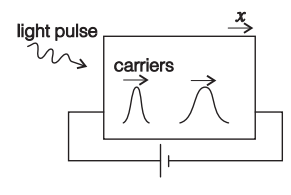

FIG. 1: Biased random walks in a disordered system (a) $A r$ rhenius cascade. Each carrier is put in random potential with a global tilt. It undergoes jumps over barriers of random heights $E_{i}$ from a metastable well to the next one on the right thanks to thermal activation. (b) Photoconductivity setup. At time $t=0$, a light pulse creates carriers in the immediate vicinity of the left electrode of an amorphous sample. The carriers of one sign then move through the sample thanks to an applied electric field. (The carriers of the opposite sign are immediately absorbed by the left electrode.)

by the Arrhenius law:

$$
\bar{\tau}_{i}=\tau_{0} e^{E_{i} / k T}
$$

where $\tau_{0}$ is a time scale, $E_{i}$ is the height of the energy barrier separating state $i$ from state $i+1, k$ is the Boltzmann constant and $T$ is the temperature. The potential global tilt is assumed to be large enough to neglect backward jumps from $i$ to $i-1$. The random walk we consider is thus completely biased. The time spent between the metastable states is neglected. For a given barrier height $E_{i}$, the lifetime distribution $\psi\left(\tau \mid E_{i}\right)$ is exponential with mean $\bar{\tau}_{i}$ :

$$
\psi\left(\tau \mid E_{i}\right)=\frac{1}{\bar{\tau}_{i}} e^{-\tau / \bar{\tau}_{i}}
$$

In the photoconductivity experiments (Fig. 10), a light pulse creates at time $t=0$ carriers localized near the surface of the sample. The carriers then move through the disordered sample thanks to an applied electric field. Thus, this situation can be modelled by a large number of Arrhenius cascade in parallel, each electron path being associated to one cascade.

One may (wrongly) expect that the transient current flowing through the sample is quasi-constant at the beginning, while the bunch of carriers propagates through the sample, before decreasing rapidly to zero when the carriers leave the sample after reaching the end electrode. But what is observed is quite different. The current decreases as a power law $\sim 1 / t^{1-\alpha}$ while the carriers are still in the sample, then as $\sim 1 / t^{1+\alpha}$ when some carriers start leaving the sample. For simplicity, we assume here that the sample is semi-infinite so that the carriers never leave the sample.

The explanation of this anomalous behaviour will be shown to be related to the distribution of lifetimes $\tau_{i}$. The randomness of the $\tau_{i}$ 's results from the combination of the exponential statistics of jump times for a given barrier height $E_{i}$ (eq. (2) ) with the barrier height statistics conveniently described by an exponential distribution $P\left(E_{i}\right)$,

$$
P\left(E_{i}\right)=\frac{1}{E_{0}} e^{-E_{i} / E_{0}} \quad \text { for } \quad E_{i} \geq 0,
$$

where $E_{0}$ is an energy scale related to the sample disorder.

The waiting time distribution $\psi(\tau)$ is then

$$
\psi(\tau)=\int_{0}^{\infty} \mathrm{d} E_{i} P\left(E_{i}\right) \psi\left(\tau \mid E_{i}\right)=\alpha \gamma\left(1+\alpha, \tau / \tau_{0}\right) \frac{\tau_{0}^{\alpha}}{\tau^{1+\alpha}}
$$

where $\gamma\left(\alpha^{\prime}, x\right)=\int_{0}^{x} e^{-u} u^{\alpha^{\prime}-1} \mathrm{~d} u$ is the incomplete gamma function and

$$
\alpha=\frac{k T}{E_{0}} .
$$

At long times, $\psi(\tau)$ tends to a power law, hence the term "fractal time random walk":

$$
\psi(\tau) \simeq \alpha^{2} \Gamma(\alpha) \frac{\tau_{0}^{\alpha}}{\tau^{1+\alpha}},
$$

with $\Gamma(\alpha)=\int_{0}^{\infty} u^{\alpha-1} e^{-u} \mathrm{~d} u$.

If $\alpha \leq 1$, states $i$ have an infinite mean lifetime $\langle\tau\rangle=\int_{0}^{\bar{\infty}} \tau \psi(\tau) \mathrm{d} \tau$. However, they are unstable since they all ultimately decay to the next state $(i+1)$. Usually, unstable states have a well defined and finite mean lifetime. Here, the somewhat paradoxical presence of unstable states with infinite mean lifetimes is at the origin of the striking properties of the fractal time random walk.

\section{B. Subrecoil laser cooling}

Laser cooling of atomic gases consists in reducing the momentum spread of atoms thanks to momentum exchanges between atoms and photons. Subrecoil laser cooling consists in reducing the momentum spread to less than a single photon momentum, denoted $\hbar k$. This paradoxical goal is achieved by introducing a momentum dependence in the photon scattering rate (see Fig. 2 2 ) so that it decreases strongly or even vanishes in the vicinity of $p=0$, where $p$ denotes the atomic momentum, taken in one dimension for simplicity.

The mechanism of subrecoil cooling is explained in Fig. 2. Any time a photon is absorbed and spontaneously reemitted by an atom, the atomic momentum undergoes a momentum kick on the order of $\hbar k$, which has a random component because spontaneous emission occurs in a random direction. Thus, the repetition of absorptionspontaneous emission cycles generates for the atom a momentum random walk (see Fig. 20), with momentum dependent waiting times $\tau$ between two kicks. When an atom reaches by chance the vicinity of $p=0$, it tends to stay there a long time. This enables to accumulate atoms at small momenta, i.e., to cool. 

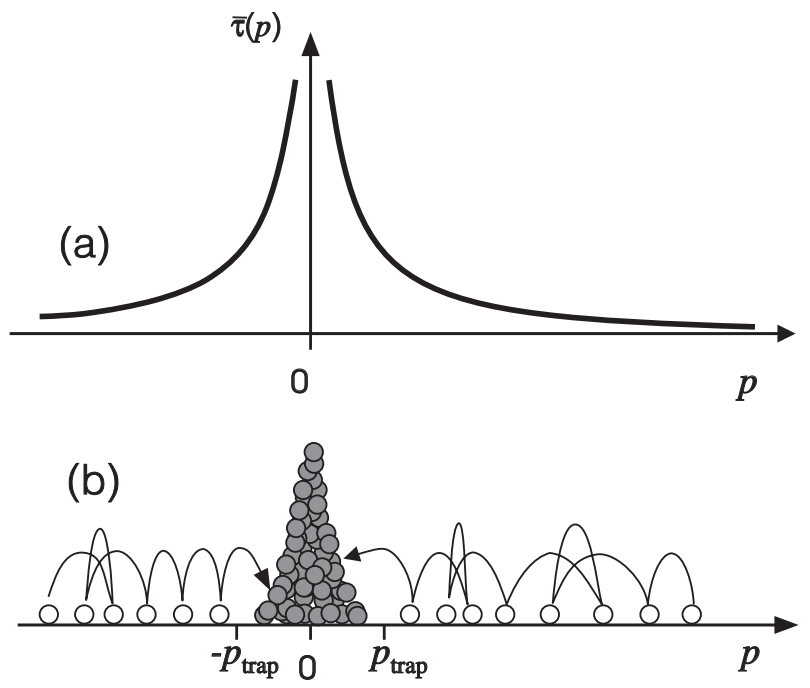

FIG. 2: Subrecoil laser cooling (a) The mean sojourn time at momentum $p \bar{\tau}(p)$ becomes very large for small atomic momenta. (b) Photon scattering creates a momentum random walk with an accumulation in the vicinity of $p=0$ due to the momentum dependence of the mean sojourn time $\bar{\tau}(p)$.

For a quantitative treatment, we introduce $\bar{\tau}(p)$, the mean sojourn time at momentum $p$ (also the mean waiting time between two spontaneous photons for an atom at momentum $p$ ). For a given $p$, the sojourn time at momentum $p$, i.e., the distribution $\psi(\tau \mid p)$ of sojourn times at momentum $p$ is

$$
\psi(\tau \mid p)=\frac{1}{\bar{\tau}(p)} e^{-\tau / \bar{\tau}(p)}
$$

We need to characterize the distribution of "landing" momenta $\pi(p)$ after a spontaneous emission. Under favourable but often realistic assumptions, atoms spend most of the time around the origin in the interval $\left[-p_{\text {trap }},+p_{\text {trap }}\right]$, because they diffuse fast outside this interval and thus come back to it rapidly after leaving it. If $p_{\text {trap }}<\hbar k$, then after a spontaneous emission, the distribution of atomic momenta can be considered as uniform:

$$
\pi(p)=\frac{1}{2 p_{\text {trap }}}
$$

The distribution of sojourn times after a spontaneous emission is thus

$$
\psi(\tau)=\int_{-p_{\text {trap }}}^{p_{\text {trap }}} \mathrm{d} p \pi(p) \psi(\tau \mid p)
$$

We consider the physically relevant case of power law $\bar{\tau}(p)$,

$$
\bar{\tau}(p)=\frac{\tau_{0} p_{0}^{\beta}}{|p|^{\beta}}
$$

where $\beta>0$, and $\tau_{0}$ and $p_{0}$ are time and momentum scales, respectively. Then, one finds, just as in the fractal time random walk, a waiting time distribution with a power law tail:

$\psi(\tau)=\frac{\alpha p_{0}}{p_{\text {trap }}} \gamma\left[1+\alpha,\left(\frac{p_{\text {trap }}}{p_{0}}\right)^{1 / \alpha} \frac{\tau}{\tau_{0}}\right] \frac{\tau_{0}^{\alpha}}{\tau^{1+\alpha}} \underset{\tau \rightarrow \infty}{\longrightarrow} \alpha^{2} \Gamma(\alpha) \frac{p_{0}}{p_{\text {trap }}} \frac{\tau_{0}^{\alpha}}{\tau^{1+\alpha}}$

where

$$
\alpha=\frac{1}{\beta} \text {. }
$$

If $\beta<1$, the mean waiting time is finite and simple integration gives

$$
\langle\tau\rangle=\frac{1}{1-\beta}\left(\frac{p_{0}}{p_{\text {trap }}}\right)^{\beta} \tau_{0} .
$$

If $\beta \geq 1$, on the contrary, the mean waiting time is infinite. This divergence of the mean has dramatic (and positive in terms of cooling) consequences (see $\coprod$ III B).

\section{Connection with renewal theory}

Renewal processes are stochastic process in which a system undergoes a sequence of events (denoted by $\bullet$ in Fig. 3) separated by independent random "waiting times" $\tau_{1}, \tau_{2}, \ldots$ The term "renewal process" comes from engineering. Assume that, at time $t=0$, one installs a machine in a factory. When, after being operated for a random lifetime $\tau_{1}$, the machine breaks down, it has to be replaced by a new one, which will work till it breaks down at $\tau_{1}+\tau_{2}$ and has to be replaced ... If, instead of a single machine, one has installed a large number of identical machines, then, to decide how many replacement machines must be stored at a given time, one needs to know the replacement rate, which we call hereafter the renewal density.

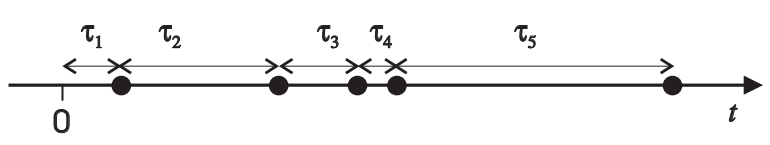

FIG. 3: Renewal processes. The system undergoes a sequence of events (jumps from trap to trap, momentum kicks ...) at random times separated by waiting times $\tau_{1}, \tau_{2}, \ldots$

To understand the statistical properties of renewal processes, various quantities are introduced. The most detailed information is provided by the distribution of the number of renewals, $f_{t}(r)$, i.e., the probability distribution for the system to undergo $r$ events in time $t$. One also introduces derived quantities, the mean number of renewals at time $t,\langle r\rangle_{t}$, and the mean renewal rate at time $t$, denoted $R(t)$ and called the renewal density. Mathematical expressions for these three quantities will be given in $\amalg \mathrm{A}$ Here we show the role they play in fractal time random walk and in subrecoil laser cooling. 
In the biased (fractal time or non fractal time) random walk, the discretized positions $n$ at time $t$ correspond directly to the number of jumps performed between time 0 and $t$. Hence the position distribution $\rho(n, t)$ in the fractal time random walk is the renewal number distribution:

$$
\rho(n, t)=f_{t}(n)
$$

The mean position of the carriers is $\langle r\rangle_{t}$. The current $i(t)$ measured in photoconductivity experiments before the carriers get out of the sample is proportional to the mean carrier velocity $\mathrm{d}\langle r\rangle_{t} / \mathrm{d} t$, which is the renewal density (see $\amalg$ ). Thus, one has

$$
i(t) \propto R(t) .
$$

In subrecoil cooling, the momentum distribution $\rho(p, t)$ can be written in the following form:

$$
\rho(p, t)=\pi(p) \int_{0}^{t} \mathrm{~d} t_{l} R\left(t_{l}\right) P_{s}\left(t-t_{l} \mid p\right),
$$

where $t_{l}$ is the time of the last jump occurring between 0 and $t, R\left(t_{l}\right) \mathrm{d} t_{l}$ is the probability that a jump occurs during the interval $\left[t_{l}, t_{l}+\mathrm{d} t_{l}\right), \pi(p)$ is the uniform probability distribution for a jumping atom to land at momentum $p$ and $P_{s}\left(t-t_{l} \mid p\right)$ is the survival probability for an atom landing at momentum $p$ at time $t_{l}$ to stay there till at least time $t$. Using eq. (7), one has trivially

$$
P_{s}\left(t-t_{l} \mid p\right)=\int_{t-t_{l}}^{\infty} \mathrm{d} \tau \psi(\tau \mid p)=e^{-\left(t-t_{l}\right) / \bar{\tau}(p)} .
$$

The non trivial physical information is contained in the renewal density $R(t)$.

The height $h(t)$ of the momentum distribution peak,

$$
h(t)=\rho(p=0, t),
$$

is proportional to $\langle r\rangle_{t}$, the mean number of jumps between 0 and $t$. Indeed, for any jump, there is a probability $\pi(p) 2 \mathrm{~d} p=\mathrm{d} p / p_{\text {trap }}$ to fall in the vicinity [ $\left.-\mathrm{d} p, \mathrm{~d} p\right]$ of the origin and to stay there indefinitely since states in $[-\mathrm{d} p, \mathrm{~d} p]$ have arbitrarily long lifetimes in the limit $\mathrm{d} p \rightarrow 0(\bar{\tau}(p) \underset{p \rightarrow 0}{\longrightarrow} \infty$, see eq. (10) $)$. Thus the height writes

$$
h(t)=\frac{\langle r\rangle_{t}}{2 p_{\text {trap }}}
$$

\section{RENEWAL THEORY AND LÉVY STABLE LAWS}

\section{A. General formulae}

The number of renewals $r_{t}$ in a time $t$ is defined as the number of jumps having occurred before time $t$. It satisfies

$$
S_{r_{t}} \leq t<S_{r_{t}+1}
$$

where $S_{r_{t}}=\sum_{i=1}^{r_{t}} \tau_{i}$ is the sum of the first $r_{t}$ waiting times. The relationship between the renewal number distribution $f_{t}(r)$ and the waiting time distribution $\psi(\tau)$ can be obtained from the following simple reasoning.

Note first that the distribution, denoted $\psi^{r *}\left(S_{r}\right)$, of the sum $S_{r}$ of $r$ independent identically distributed waiting times is the $r^{\text {th }}$ convolution product of $\psi(\tau)$ with itself. Moreover, from the definition of the number $r_{t}$ of renewals, one has obviously

$$
\operatorname{Pr}\left(r_{t}<r\right)=\operatorname{Pr}\left(S_{r}>t\right)=\left[1-\Psi^{r *}(t)\right],
$$

where $\Psi^{r *}\left(S_{r}\right)=\int_{0}^{S_{r}} \psi^{r *}(u) \mathrm{d} u$ denotes the distribution function of $S_{r}$ (in spite of its notation, $\Psi^{r *}\left(S_{r}\right)$ is not the $r^{\text {th }}$ convolution product of the waiting time distribution function $\left.\Psi(\tau)=\int_{0}^{\tau} \psi(u) \mathrm{d} u\right)$. The probability distribution $f_{t}(r)$ of the number of renewals at time $t$ is thus finally

$f_{t}\left(r_{t}=r\right)=\operatorname{Pr}\left(r_{t}<r+1\right)-\operatorname{Pr}\left(r_{t}<r\right)=\Psi^{r *}(t)-\Psi^{(r+1) *}(t)$.

This expression relates the distribution of a discrete random variable, $r$, to the distribution fonctions of continuous random variables $t$. Important quantities derived from the renewal number distribution $f_{t}(r)$ are the mean number of renewals at time $t$ :

$$
\langle r\rangle_{t}=\sum_{r=0}^{\infty} r f_{t}(r)
$$

and the renewal density, i.e., the mean number of renewals per unit time 19$]$ :

$$
R(t)=\frac{\mathrm{d}\langle r\rangle_{t}}{\mathrm{~d} t}
$$

Usual theoretical treatments of renewal problems are based on Laplace transforms of the waiting time distribution that are indeed well suited to handle the convolution products $\psi^{r *}(t)$. Here, we prefer a different approach based only on the generalized central limit theorem. This approach requires nearly no calculation. Moreover, it stresses the role of the behaviour of sums of many random variables.

Indeed, most useful distributions tend, under repeated convolution, to a Lévy stable law given by the generalized central limit theorem. Thus we can obtain from eq. (22) analytical expressions for $f_{t}(r)$ and for related quantities in the limit of large $r$ and hence large $t$. Depending on the finiteness of the first two moments of the waiting time distribution $\psi(\tau)$, three cases can be distinguished. For brevity, we treat only the two most striking cases: first and second moment both finite in $\oiiint$ first and second moment both infinite in $\$$ IIC

\section{B. Case of waiting time distributions with finite first and second moment}

When both the mean $\mu=\langle\tau\rangle$ and the variance $\sigma^{2}$ of the waiting time are finite, the (usual) central limit the- 
orem applies and $\psi^{r *}(t)$ tends to a Gaussian distribution

$$
\psi^{r *}(t) \underset{r \rightarrow \infty}{\longrightarrow} \frac{1}{\sqrt{2 \pi} \sigma_{r}} \exp \left[-\frac{\left(t-\mu_{r}\right)^{2}}{2 \sigma_{r}^{2}}\right]
$$

with mean $\mu_{r}=r \mu$ and variance $\sigma_{r}^{2}=r \sigma^{2}$. Thus, after changing variables in eq. (22), one has

$$
f_{t}(r) \underset{r \rightarrow \infty}{\longrightarrow} \int_{\left(t-\mu_{r+1}\right) / \sigma_{r+1}}^{\left(t-\mu_{r}\right) / \sigma_{r}} \frac{1}{\sqrt{2 \pi}} e^{-u^{2} / 2} \mathrm{~d} u .
$$

Fix $\left(t-\mu_{r}\right) / \sigma_{r}$ and take $t \rightarrow \infty$. One expects intuitively that the number of renewals is approximately given by $t / \mu \gg 1$ at large $t$ (this is validated a posteriori by eq. (28) $)$. Thus $\left(t-\mu_{r}\right) / \sigma_{r} \simeq(t-\mu r) /(\sigma \sqrt{t / \mu})$ and $\left(t-\mu_{r+1}\right) / \sigma_{r+1} \simeq(t-\mu r) /(\sigma \sqrt{t / \mu})-\sqrt{\frac{\mu^{3}}{\sigma^{2} t}}$. This leads for the number of renewals to a Gaussian distribution with mean $t / \mu$ and variance $\sigma^{2} t / \mu^{3}$ :

$$
f_{t}(r) \underset{t \rightarrow \infty}{\longrightarrow} \frac{1}{\sqrt{2 \pi \sigma^{2} t / \mu^{3}}} \exp \left[-\frac{(r-t / \mu)^{2}}{2 \sigma^{2} t / \mu^{3}}\right] .
$$

Although well known in renewal theory, this result is non trivial: the distribution of the sums of $r(\rightarrow \infty)$ terms and of the number of renewals at large times are found to have the same (Gaussian) shape. This is grossly violated with infinite mean waiting times (see \$IC).

As direct consequences of eq. (27), the mean number of renewals $\langle r\rangle_{t}$ tends to $t / \mu$ at large times:

$$
\langle r\rangle_{t} \underset{t \rightarrow \infty}{\longrightarrow} \frac{t}{\mu}
$$

and, using eq. (24), the renewal density tends to the reciprocal of the mean waiting time:

$$
R(t) \underset{t \rightarrow \infty}{\longrightarrow} \frac{1}{\mu},
$$

in agreement with intuition.

Physical comments and an example of a renewal process with finite mean waiting time will be presented in IIIA

\section{Case of waiting time distributions with infinite first and second moment}

We consider now the case of waiting time distributions with infinite first two moments, focusing on the canonical example of distributions with (Pareto) power law tails of index $\alpha$,

$$
\psi(\tau) \underset{\tau \rightarrow \infty}{\longrightarrow} \frac{\alpha \tau_{0}^{\alpha}}{\tau^{1+\alpha}},
$$

with $0<\alpha<1$ (slightly more general cases can be handled using the theory of regular variations). The distributions $\psi^{r *}$ of the sums $S_{r}$ no longer tends to Gaussians but, according to the generalized central limit theorem, to Lévy stable laws:

$$
\psi^{r *}(t) \underset{r \rightarrow \infty}{\longrightarrow} \frac{1}{r^{1 / \alpha}} L_{\alpha, B}\left(\frac{t}{r^{1 / \alpha}}\right)
$$

where $L_{\alpha, B}(u)$ is a one-sided $(u \geq 0)$ Lévy stable law given by its Laplace transform,

$$
\int_{0}^{\infty} e^{-s u} L_{\alpha, B}(u) \mathrm{d} u=e^{-B s^{\alpha}}
$$

and $B$ is a scale parameter given by

$$
B=\Gamma(1-\alpha) \tau_{0}^{\alpha}
$$

After changing variables in eq. (22), one obtains

$$
f_{t}(r)=\int_{t /(r+1)^{1 / \alpha}}^{t / r^{1 / \alpha}} L_{\alpha, B}(u) \mathrm{d} u
$$

which leads to the following asymptotic expression [20]:

$$
f_{t}(r) \underset{t \rightarrow \infty}{\longrightarrow} \frac{t}{\alpha r^{1+1 / \alpha}} L_{\alpha, B}\left(\frac{t}{r^{1 / \alpha}}\right)
$$

In sharp contrast with the finite moments case, the renewal number distribution $f_{t}(r)$ differs strongly from the distribution of the sum (eq. (31) ) even though both distributions involve Lévy stable laws. As will be shown in ஹIIA $f_{t}(r)$ has a slow decay at small $r$ and a fast decay at large $r$ unlike $\psi^{r *}(t)$ which decays as $1 / t^{1+\alpha}$.

The mean number of renewals $\langle r\rangle_{t}$ is finite and can be related to a negative moment of a Lévy stable law:

$$
\langle r\rangle_{t} \simeq \int_{0}^{\infty} r f_{t}(r) \mathrm{d} r=t^{\alpha} \int_{0}^{\infty} u^{-\alpha} L_{\alpha, B}(u) \mathrm{d} u .
$$

Hence, using $\int_{0}^{\infty} u^{-\alpha} L_{\alpha, B}(u) \mathrm{d} u=1 /(B \alpha \Gamma(\alpha))$ and eq. (33), one has finally

$$
\langle r\rangle_{t} \underset{t \rightarrow \infty}{\longrightarrow} \frac{\sin (\pi \alpha)}{\pi \alpha}\left(\frac{t}{\tau_{0}}\right)^{\alpha} .
$$

Finally, using eq. (24), the renewal density tends asymptotically to an ever decreasing power law:

$$
R(t) \underset{t \rightarrow \infty}{\longrightarrow} \frac{\sin (\pi \alpha)}{\pi} \frac{1}{\tau_{0}^{\alpha} t^{1-\alpha}} .
$$

Physical comments and an example of a renewal process with infinite mean waiting time will be presented in III A

\section{APPLICATION TO FRACTAL TIME RANDOM WALK AND SUBRECOIL COOLING}

We have seen in $\$ \mathrm{C}$ that the most important quantities appearing in biased random walks and in subrecoil cooling are directly related to renewal theory: renewal number distribution $f_{t}(r)$ (position distribution 
in biased random walks), mean number of renewals $\langle r\rangle_{t}$ (peak height in subrecoil cooling) and renewal density $R(t)$ (current in the biased random walk, momentum distribution in subrecoil cooling).

In this section, we thus apply the results on renewal processes obtained in $\$$ to biased random walks and subrecoil cooling. We emphasize the physics consequences of the divergence of the mean waiting time in renewal processes by opposing, for each application, one example with finite mean waiting time and one example with infinite mean waiting time. These examples reveal the generic features of the cases with finite or infinite mean waiting times.

\section{A. Biased random walks}

As an example of waiting time distribution with finite mean and standard deviation generating a (non fractal time) random walk, we consider an exponential distribution

$$
\psi(\tau)=\frac{1}{\mu} e^{-\tau / \mu} \quad \text { for } \quad \tau \geq 0
$$

with mean and standard deviation both equal to $\mu$. In this case, the convolutions of $\psi(\tau)$ have the simple explicit forms of the Gamma (or Erlang) distributions

$$
\psi^{n *}(\tau)=\frac{\tau^{n-1}}{(n-1) ! \mu^{n}} e^{-\tau / \mu} .
$$

Thus, applying eq. (22), the renewal density $f_{t}(r)$ and hence the position distribution $\rho(n, t)$ are exactly known:

$$
\rho(n, t)=\frac{e^{-t / \mu}}{n !}\left(\frac{t}{\mu}\right)^{n} .
$$

(One recognizes the Poisson distribution of mean $\langle r\rangle_{t}=$ $t / \mu$, as expected: in this case, the renewal process is a Poisson process.) As shown in Fig. [4 this exact position distribution rapidly tends to the Gaussian distribution given by eq. (27):

$$
\rho(n, t) \underset{t \rightarrow \infty}{\longrightarrow} \frac{1}{\sqrt{2 \pi t / \mu}} \exp \left[-\frac{(n-t / \mu)^{2}}{2 t / \mu}\right] .
$$

We thus recover the intuitive picture of normal transport: a position distribution propagating at a constant speed $1 / \mu$ and spreading as $\sqrt{t}$. The current $i(t)$ resulting from such a distribution in photoconductivity experiments is related to the renewal density (eq. (15)) which leads, using eq. (29), to

$$
i(t) \propto 1 / \mu .
$$

As an example of waiting time distribution with infinite mean and standard deviation generating a fractal

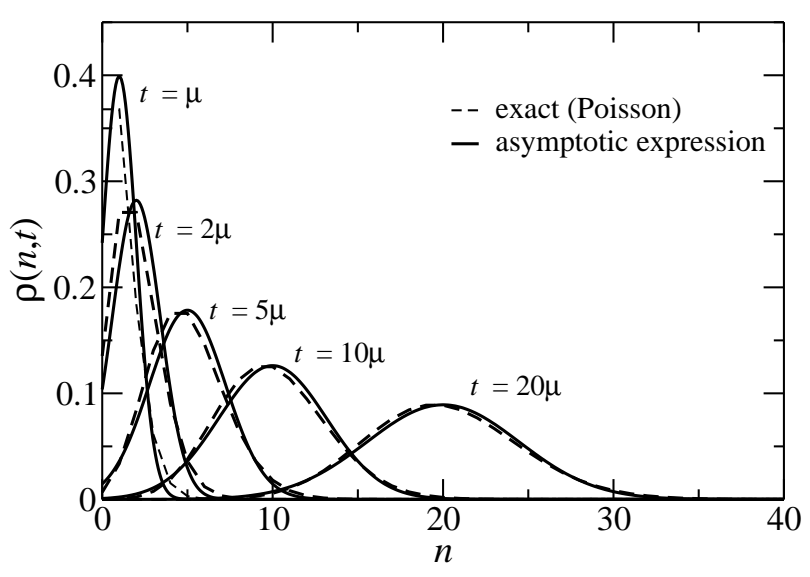

FIG. 4: Time evolution of the position distribution $\rho(n, t)$ for a biased random walk with an exponential waiting time distribution (finite $\langle\tau\rangle$ ). The distribution propagates at constant speed $1 / \mu$ and spreads as $\sqrt{t}$. The exact result (eq. (41)) and the asymptotic one (eq. (42) agree even at short times.

time random walk, we consider a Pareto distribution 21] of index $\alpha=1 / 2$,

$$
\psi(\tau)=\frac{\tau_{0}^{1 / 2}}{2 \tau^{3 / 2}} \quad \text { for } \quad \tau \geq \tau_{0}
$$

with $\tau_{0}>0$. As there is no simple analytic form for $\psi^{n *}(t)$ and thus for the position distribution $\rho(n, t)=$ $\Psi^{n *}(t)-\Psi^{(n+1) *}(t)$ (eq. (22)), we obtain $\rho(n, t)$ through numerical simulation (see Fig. 5). On the other hand, using eq. (35) and the known form for the asymmetric Lévy stable law of index $1 / 2$ called the Smirnov law (or using eq. (32) ), the asymptotic distribution is found to be a half-Gaussian

$$
\rho(n, t) \underset{t \rightarrow \infty}{=} \sqrt{\frac{\tau_{0}}{t}} \exp \left(-\frac{\pi \tau_{0}}{4 t} n^{2}\right) .
$$

The fact that a half-Gaussian is obtained should not give the impression that fractal time transport is similar to normal transport described by full Gaussians (eq. (42)). First, the half-Gaussian is specific to Pareto waiting time distributions with $\alpha=1 / 2$ (see below for other $\alpha$ 's). Second, the properties of the half-Gaussian in fractal time transport are completely different from those of the full Gaussian of the normal transport. Indeed, instead of propagating, the distribution peak remains at the origin $n=0$ at all times. Only the tails spread to the right, more and more slowly as times goes by. This is due to the fact that the carriers statistically tend to be trapped into deeper and deeper traps at long times, which slows down their motion. The resulting current is thus a decreasing function of time, at all times. Using eq. (15) and 
eq. (38), one obtains:

$$
i(t) \sim \frac{1}{\sqrt{\tau_{0} t}}
$$

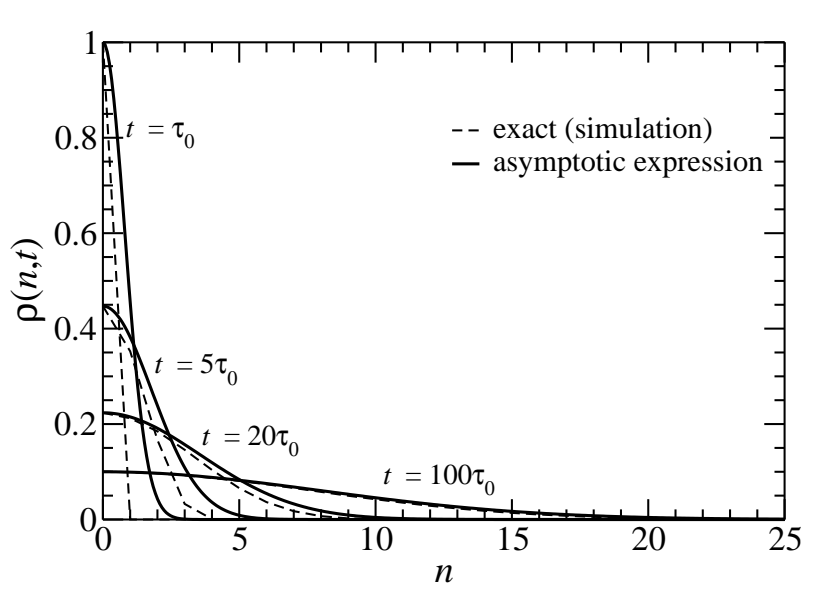

FIG. 5: Time evolution of the position distribution $\rho(n, t)$ for a biased fractal time random walk constructed from a Pareto distribution of index $\alpha=1 / 2$ (infinite $\langle\tau\rangle$ ). The distribution spreads slowly towards positive values but its maximum always remains at the origin. Note the smaller position scale and the longer time scales compared to Fig. 4 which emphasizes the slowness of transport in the fractal time random walk. The exact (simulated) and asymptotic results (eq. (45)) are in good agreement. (They have seemingly different norms at short times because the exact result is a discrete distribution while the asymptotic one is a continuous distribution.)

It is worth examining the general case of waiting time distributions with power law tails like eq. (30) and infinite means $(\alpha<1)$. From the following expansion of asymmetric Lévy stable laws with $\alpha<1$,

$$
L_{\alpha, B}(x) \underset{x \rightarrow \infty}{\simeq} \frac{\alpha B}{\Gamma(1-\alpha) x^{1+\alpha}},
$$

one finds the behaviour close to the origin (for $t \rightarrow \infty$ ):

$$
\rho(n, t) \underset{n \rightarrow 0}{\simeq}\left(\frac{\tau_{0}}{t}\right)^{\alpha} .
$$

Hence, the position distribution is flat ( $n$ independent) at small $n$, as already observed for the special case $\alpha=1 / 2$. Moreover, using

$$
L_{\alpha, B}(x) \underset{x \rightarrow 0}{\simeq} A x^{\frac{\alpha-2}{2(1-\alpha)}} \exp \left[-\frac{C}{x^{\alpha /(1-\alpha)}}\right],
$$

where $A$ and $C$ are constants, one obtains

$$
\rho(n, t) \propto \exp \left(-\frac{C r^{1 /(1-\alpha)}}{t^{\alpha /(1-\alpha)}}\right)
$$

up to power law corrections. As $1 /(1-\alpha)>1$, the transport front always decreases faster than exponentially and presents a well defined characteristic position. Consequently, the mean carrier position yielding the current, is well defined, unlike the mean waiting time which is infinite. Using eq. (15) and eq. (38), one finds that the current decays as a power law:

$$
i(t) \propto \frac{1}{t^{1-\alpha}},
$$

as already observed for the special case $\alpha=1 / 2$ and in agreement with photoconductivity transient experiments.

\section{B. Subrecoil cooling}

Consider first the case of waiting time distributions with $\alpha=1 / \beta>2$ (eq. (11)) ensuring a finite mean waiting time $\langle\tau\rangle$ (and a finite $\left\langle\tau^{2}\right\rangle$ ). According to eq. (29) and eq. (13), one has

$$
R(t) \underset{t \rightarrow \infty}{\longrightarrow} \frac{1-\beta}{\tau_{0}}\left(\frac{p_{\text {trap }}}{p_{0}}\right)^{\beta}
$$

and thus, applying eq. (16) with eq. (8) and eq. (17), one finds the momentum distribution

$$
\rho(p, t)=\frac{1-\beta}{2 p_{\text {trap }}}\left[1-e^{-\frac{t}{\tau_{0}}\left(\frac{|p|}{p_{0}}\right)^{\beta}}\right]\left(\frac{p_{\text {trap }}}{|p|}\right)^{\beta} .
$$

This distribution has stationary tails:

$$
\rho(p, t) \simeq \frac{1-\beta}{2 p_{\text {trap }}}\left(\frac{p_{\text {trap }}}{|p|}\right)^{\beta} \text { for }|p|>p_{0}\left(\frac{\tau_{0}}{t}\right)^{1 / \beta}
$$

but a non stationary peak that increases linearly in time:

$$
\rho(p, t) \simeq \frac{1-\beta}{2 p_{0}^{\beta} p_{\text {trap }}^{1-\beta}} \frac{t}{\tau_{0}} \quad \text { for } \quad|p|<p_{0}\left(\frac{\tau_{0}}{t}\right)^{1 / \beta} .
$$

This peak is also obtained directly using relation (19) between the height $h(t)$ and the mean number of renewals of eq. (28).

Finite mean waiting times are not very favourable for the cooling since only a vanishingly small fraction of atoms goes on accumulating at smaller and smaller velocities (see Fig. [6).

Consider now the case of waiting time distributions with infinite mean waiting times $(\alpha=1 / \beta<1)$ ensuring. According to eq. (38), one has

$$
R(t) \underset{t \rightarrow \infty}{\longrightarrow} \frac{\sin (\pi \alpha) p_{\text {trap }}}{\pi \alpha \Gamma(\alpha) p_{0}} \frac{1}{\tau_{0}^{\alpha} t^{1-\alpha}}
$$

(one must write $\psi(t)$ of eq. (11) in the form of eq. (30), thus replacing $\tau_{0}^{\alpha}$ by $\alpha \Gamma(\alpha)\left(p_{0} / p_{\text {trap }}\right) \tau_{0}^{\alpha}$ in eq. (38)). 


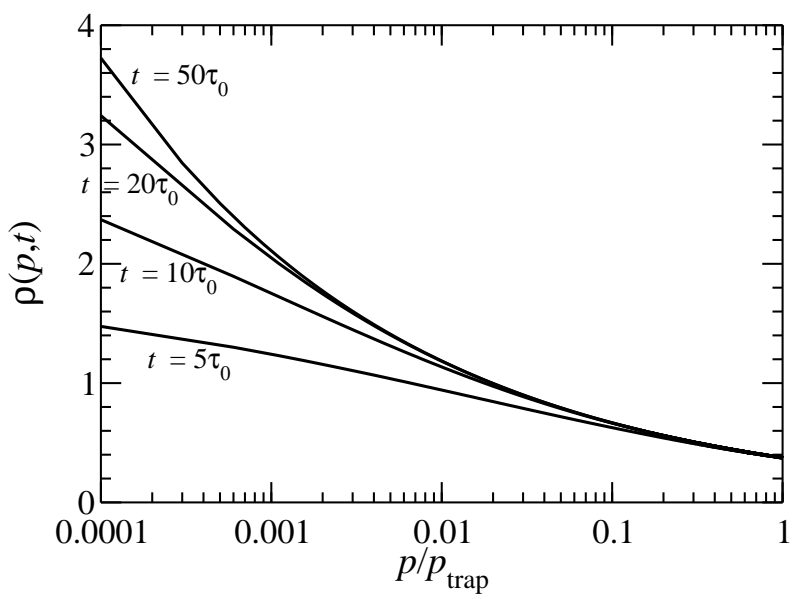

FIG. 6: Time evolution of the momentum distribution $\rho(p, t)$ for subrecoil cooling with a finite mean waiting time. Parameters: $\beta=0.25, p_{0}=1$. The tails reach a stationary state. Only a vanishingly narrow part of the peak goes on increasing at long times (note the logarithmic $p$ scale).

Thus, applying eq. (16) with eq. (8) and eq. (17), the momentum distribution writes

$$
\rho(p, t) \underset{t \rightarrow \infty}{\longrightarrow} \frac{\sin (\pi \alpha)}{2 \pi \alpha^{2} \Gamma(\alpha) p_{0}}\left(\frac{t}{\tau_{0}}\right)^{\alpha} \mathcal{G}\left(\frac{t p^{\beta}}{\tau_{0} p_{0}^{\beta}}\right),
$$

where

$$
\mathcal{G}(q)=\alpha \int_{0}^{1} \mathrm{~d} u u^{\alpha-1} e^{-(1-u) q}
$$

is a confluent hypergeometric function. Thus $\rho(p, t)$ presents a scaling form and evolves at all times scales, with no stationary state. The tails behave as

$\rho(p, t) \underset{t \rightarrow \infty}{\longrightarrow} \frac{\sin (\pi \alpha)}{2 \pi \alpha \Gamma(\alpha)}\left(\frac{\tau_{0}}{t}\right)^{1-\alpha} \frac{1}{p_{0}^{1-\beta} p^{\beta}}$ for $|p|>p_{0}\left(\frac{\tau_{0}}{t}\right)^{1 /}$

and the peak, also obtained directly from eq. (19) and the mean number of renewals (37), behaves as

$\rho(p, t) \underset{t \rightarrow \infty}{\longrightarrow} \frac{\sin (\pi \alpha)}{2 \pi \alpha^{2} \Gamma(\alpha) p_{0}}\left(\frac{t}{\tau_{0}}\right)^{\alpha}$ for $|p|<p_{0}\left(\frac{\tau_{0}}{t}\right)^{1 / \beta}$

Infinite mean waiting times are favourable for the cooling (Fig. 7): all atoms accumulate in a narrower and narrower peak in the vicinity of $p=0$. The cooling goes on without fundamental limits at long times. The absence of limits is related to the significant weight $(c f .\langle\tau\rangle=\infty)$ of $p$ states with arbitrarily long lifetimes.

\section{BIBLIOGRAPHICAL NOTES}

Fractal time The theory of fractal time random walks was developed in particular cases in [7] using continu-

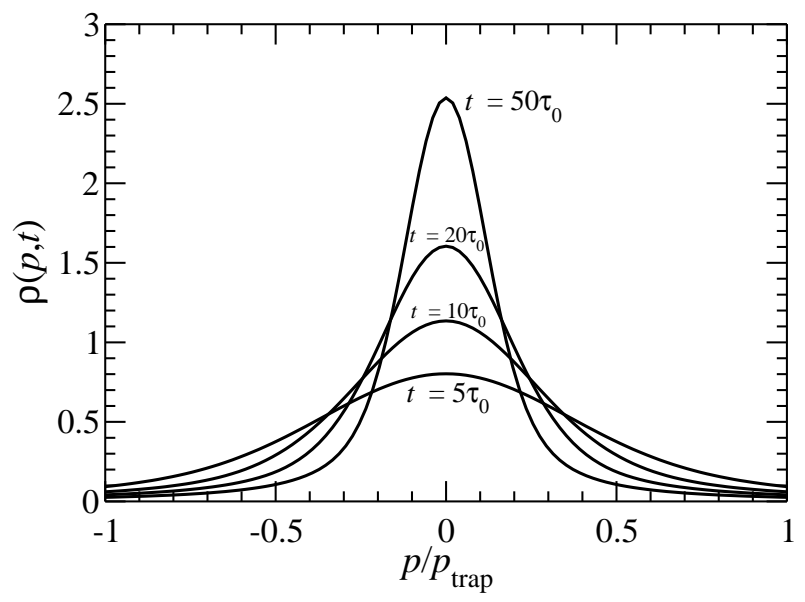

FIG. 7: Time evolution of the momentum distribution $\rho(p, t)$ for subrecoil cooling with an infinite mean waiting time. Parameters: $\beta=2, p_{0}=1$. The full momentum distribution, tails and peak, never reaches a stationary state. All atoms go on accumulating without limit in a narrower and narrower peak as time increases.

ous time random walks and, in the general case, in [1], adding Tauberian techniques to handle Laplace transforms. Fractal times were used in [8] to model turbulent diffusion. Several other applications involving fractal times were presented in [9] and [2].

Subrecoil cooling The statistical approach to subrecoil cooling was developed in three steps: 10], 11] and [4]. The connection of this approach with renewal theory was stressed in [5].

Renewal processes The basic theory of renewal processes is presented in a small book [12]. This book does not include infinite mean waiting times but some general 1/expressions for finite mean waiting times are still valid in this case too (see $[$ IIA). Ref. 13], chapter XI, presents renewal theory with a more theoretical viewpoint and includes some results on infinite mean waiting times which were discovered at the beginning of the sixties, in spite of the fact that no application seemed to be known at the time. Renewal processes in a physics context including infinite mean waiting times have been studied systematically in 14] using Laplace transform techniques.

The mean number of renewals given by eq. (37) for infinite mean waiting times agrees with eq. (8) in [1] obtained through Tauberian theorems for the fractal time random walk (there is to be a misprint in this reference: the expression for $\psi(\tau)$, page 424 , line 3 , must be replaced by $\left.\psi(\tau) \sim \alpha /\left[t^{1+\alpha} \Gamma(1+\alpha) A(t)\right]\right)$. It also agrees with the renewal theory developed in [14] (eq. (3.6)).

The renewal number distribution expressed with Lévy stable laws given by eq. (35) agrees with eq. (5.6) of [13], which is however more complicated.

Generalized central limit theorem The generalized central limit theorem and results on Lévy stable laws 
used in $₫ \mathrm{C}$ can be found, e.g. in Appendix B of [15] or in $\S 4.2$ of 4 ]. Details on Lévy stable laws can also be found in $\S 1.2$ of [16].

\section{Conclusion}

This paper has underlined the common statistical core at work in two seemingly opposite problems: a diffusion mechanism (fractal time random walk) and a cooling mechanism (subrecoil laser cooling). This core is made of waiting time distributions with infinite means.

Usual theoretical techniques for these problems are based on Tauberian theorems which, through the Laplace transform, imply some loss of physical intuition. Here, we have presented a renewal theory approach which, thanks to the generalized central limit theorem, provides a shortcut to obtain physically relevant quantities. It emphasizes the key contribution of the unusual behaviour of sums of random variables with infinite means.

Understanding these stochastic processes with infinite means is not a purely academic game. It has already led to significant improvements of laser cooling strategies [17] and other improvements are under way [18].

\section{Acknowledgments}

I thank O.E. Barndorff-Nielsen, M. Romeo and M. Shlesinger for discussions.
[1] M. F. Shlesinger, J. Stat. Phys. 10, 421 (1974).

[2] H. Scher, M. F. Shlesinger, and J. T. Bendler, Physics Today pp. 26-34 (1991).

[3] A. Aspect, E. Arimondo, R. Kaiser, N. Vansteenkiste, and C. Cohen-Tannoudji, Phys. Rev. Lett. 61, 826 (1988).

[4] F. Bardou, J.-P. Bouchaud, A. Aspect, and C. CohenTannoudji, Lévy Statistics and Laser Cooling (Cambridge University Press, Cambridge, 2002).

[5] O. E. Barndorff-Nielsen and F. E. Benth, in State of the Art in Probability and Mathematical Statistics; Festschrift for Willem $R$. van Zwet, edited by M. C. M. de Gunst, C. A. J. Klaassen, and A. W. van der Vaart (Inst. Mathematical Statistics, 2000), Lecture Notes - Monograph Series, pp. 50-71, http://www.maphysto.dk/cgi-bin/w3$\mathrm{msql} /$ publications/genericpublication.html?publ=159.

[6] F. Bardou, in Lévy processes: theory and applications, edited by O. E. Barndorff-Nielsen, S. E. Graversen, and T. Mikosh (MaPhySto, Aarhus, 1999), Miscillanea no. 11, http://arxiv.org/abs/physics/0012049.

[7] E. W. Montroll and H. Scher, J. Stat. Phys. 9, 101 (1973).

[8] M. F. Shlesinger, B. J. West, and J. Klafter, Phys. Rev. Lett. 58, 1100 (1987).

[9] M. F. Shlesinger, Ann. Rev. Phys. Chem. 39, 269 (1988).

[10] F. Bardou, J.-P. Bouchaud, O. Emile, A. Aspect, and C. Cohen-Tannoudji, Phys. Rev. Lett. 72, 203 (1994).

[11] F. Bardou, Ph.d. thesis (1995).

[12] D. R. Cox, Renewal Theory (Methuen (Wiley), London
(New York), 1962).

[13] W. Feller, An introduction to probability theory and its Applications. Volume II (John Wiley and sons, New York, 1971).

[14] C. Godrèche and J. M. Luck, J. Stat. Phys. 104, 489 (2001).

[15] J.-P. Bouchaud and A. Georges, Phys. Rep. 195, 127 (1990).

[16] G. Samorodnitsky and M. S. Taqqu, Stable non-Gaussian random processes (Chapman \& Hall/CRC, Boca Raton, 1994).

[17] J. Reichel, F. Bardou, M. B. Dahan, E. Peik, S. Rand, C. Salomon, and C. Cohen-Tannoudji, Phys. Rev. Lett. 75, 4575 (1995).

[18] F.Bardou, Communication at the Workshop on Aspects of Large Quantum Systems Related to Bose-Einstein Condensation (Aarhus, April 2004, and to be published, 2004).

[19] The renewal density is a rate of events. It has the same dimension as a probability distribution of times, $1 /$ time, but it is not a probability density. Thus, its integral $\int_{0}^{\infty} \mathrm{d} t R(t)$, representing the average total number of events occurring between $t=0$ and $t=\infty$ does not have to be normalized. It is actually often infinite.

[20] As $t \simeq S_{r}=\sum_{i=1}^{r} \tau_{i}$ scales as $r^{1 / \alpha}$, the limit $r \rightarrow \infty$ corresponds to the limit $t \rightarrow \infty$.

[21] For a Pareto distribution of index $\alpha>2$ (finite standard deviation), the normal transport case is recovered. 